\title{
COMPARACIÓN DE LAS INTELIGENCIAS MÚLTIPLES EN NIÑOS(AS) QUE PERTENECEN A ESCUELAS CON DISTINTOS MODELOS PEDAGÓGICOS
}

\author{
Lic. Ariana Serrano Madrigal \\ Escuela Ciencias del Deporte, Universidad Nacional \\ anairaonarres@gmail.com \\ 2007
}

\section{RESUMEN}

El propósito de esta investigación fue aplicar un test que mida las distintas inteligencias múltiples en niños(as) de dos distintas escuelas, para así valorar si existe diferencia entre el modelo pedagógico academicista (enfoque tradicional) establecido por el Ministerio de Educación Pública y el modelo pedagógico cognitivo (MPC) (enfoque constructivista). En este estudio participaron 29 niños y 20 niñas, con edades entre los 8 y 12 años, pertenecientes a dos distintas escuelas públicas de Heredia (Escuela Laboratorio y Escuela de San Isidro). El instrumento que se utilizó fue un Test de Inteligencias Múltiples para niños(as) en edades escolares (Vega, 2006), el cual consta de 15 ítems, subdivididos en siete categorías: lingüística, lógico-matemática, visual, cinestésica, musical, interpersonal e intrapersonal. Para el análisis de datos se utilizó la estadística descriptiva y la estadística inferencial (ANOVA de dos vías). Se encontraron diferencias significativas en la inteligencia lingüística $(F: 9,47 ; p<0,01)$ entre escuelas MPC (3,24 $\pm 1,24$ puntos) y academicista $(2,31 \pm 1,10$ puntos), asimismo se encontraron diferencias entre sexo ( $F$ : 5,26; $p<$ $0,05)$, mujeres $(3,25 \pm 1,02$ puntos) y hombres $(2,52 \pm 1,30$ puntos). Además, en la inteligencia musical se encontraron diferencias estadísticamente significativas entre sexo (F: 7,97; $p<0,05)$. Se puede concluir que los modelos pedagógicos de enseñanza de los centros educativos públicos de Costa Rica deben actualizarse de acuerdo a las nuevas tendencias educativas.

PALABRAS CLAVES: modelos pedagógicos, inteligencias múltiples, teoría del aprendizaje significativo, educación, enseñanza.

\section{COMPARISON OF MULTIPLE INTELLIGENCES IN CHILDREN FROM SCHOOLS WITH DIFFERENT PEDAGOGICAL MODELS}

\section{ABSTRACT}

The purpose of this research was to apply a test that measures different multiple intelligences in children from two different elementary schools to determine whether there are differences between the Academicist Pedagogical Model (traditional approach) established by the Costa Rican Ministry of Public Education and the Cognitive Pedagogical Model (MPC) (constructivist approach). A total of 29 boys and 20 girls with ages 8 to 12 from two different public schools in Heredia (Laboratorio School and San Isidro School) participated in this study. The instrument used was a Multiple Intelligences Test for school age children (Vega, 2006), which consists of 15 items subdivided in seven categories: linguistic, logical-mathematical, visual, kinaesthetic, musical, interpersonal, and intrapersonal. Descriptive and inferential statistics (Two-Way ANOVA) were used for the analysis of data. Significant differences were found in linguistic intelligence $(F: 9.47 ; p<0.01)$ between the MPC school (3.24 \pm 1.24 points) and the academicist school $(2.31 \pm 1.10$ points $)$. Differences were also found between sex $(F: 5.26 ; p<0.05)$, for girls $(3.25 \pm 1.02$ points $)$ and boys (2.52 \pm 1.30 points). In addition, the musical intelligence showed significant statistical differences between sexes (F: $7.97 ; p<$ 0.05). In conclusion, the learning pedagogical models in Costa Rican public schools must be updated based on the new learning trends.

KEY WORDS: pedagogical models, multiple intelligences, significant learning theory, education, teaching. 


\section{INTRODUCCIÓN}

Según Bolaños y Molina (1990) y Ruiz (2000) la educación es todo tipo de conocimientos: informaciones globales, comportamientos de socialización, saberes empíricos y aspectos intuitivos que un sujeto recopila a lo largo de su vida y con los cuales le es posible construir experiencias. Lo que la caracteriza es que no existe la necesidad de sistematizar el conjunto de conocimientos que la conforman y puede desarrollarse libremente y sin limitación, ni por exceso ni por defecto.

La educación no es sino la vida o en el medio que conduce al ser humano, a ser inteligente, racional y consiente, a es ejercitar, desarrollar y manifestar los elementos de vida que posee por si propio (Fröbel, 1928).

Flinter (1972) expresa que la educación no puede formar al ser humano de un modo uniforme bajo la presión de ideales absolutos, no puede ser idealista. Su objetivo deber ser que la persona aprenda a ayudarse así misma, para comprender en su vida lo auténticamente humano, hacerlo suyo y salir en cualquier situación que se le presente.

Es por esto que Brenes y Porras (1994) afirman que enseñar se deriva etimológicamente de insignare, cuyo significado es "enseñar en". Atendiendo a su origen, esta es la acción de señalar contenidos para que el aprendiz se fije y pueda activamente asimilar lo que le indican.

Para Ruiz (2000), la enseñanza es una práctica sistematizada del quehacer educativo que posibilita el desarrollo del pensamiento, institucionaliza los aspectos educativos formales, constituye la manera y los momentos cómo y en los cuales los y las estudiantes se apropian del conocimiento.

En adicción Coll (1988) citado por Díaz-Barriga y Hernández (2002) menciona que la finalidad de la intervención pedagógica es desarrollar en el alumnado la capacidad de realizar aprendizajes significativos por si solos en una amplia gama de situaciones y circunstancias (aprender a aprender).

Rodríguez y Muñoz (2000) mencionan que la función de todo docente, está caracterizada por tres aspectos vitales: un carácter laboral porque ésta es la vía fundamental de la satisfacción de las necesidades y de la transformación del ser humano; la parte investigativa, porque en lo científica se ofrece el instrumento, la metodología para resolver los problemas haciendo más eficiente su labor y el ser académico, ya que el estudiante para su educación necesita apropiarse de parte de la cultura de la humanidad, en ocasiones de un modo abstracto.

Sin dejar de lado al estudiante, Hernández (1998) menciona que el docente debe conocer con profundidad su desarrollo integral, sus motivaciones y condiciones socioculturales, con el fin de que se pueda organizar los procesos de enseñanza y aprendizaje necesarios para favorecer su desarrollo pleno y autónomo.

No obstante Zapata (1989), afirma que la educación intelectualizada impide y bloquea toda manifestación espontánea y libre de la infancia e incluso con frecuencia, los educadores convierten a los juegos infantiles en meros instrumentos de instrucción, 
provocando así la pérdida de aspectos como el ser pleno en alegría y en completa libertad.

Según Armstrong (2001), uno de los inconvenientes más graves de las escuelas es su falta de flexibilidad a la hora de enseñar una materia o habilidad práctica. Los maestros(as) presentan el material de cierta forma y si los escolares no comprenden, es problema de los niños(as), no del profesor(a).

Cuando se analizan los programas de enseñanza se puede observar que se limitan a concentrarse en el predominio de las inteligencias lingüística y matemática dando mínima importancia a las otras posibilidades del conocimiento. Aquí, el por qué, muchos estudiantes no se destacan en el dominio de las inteligencias académicas tradicionales, no tienen reconocimiento y se diluye así su aporte al ámbito cultural, social y hasta se piensa que han fracasado, cuando en realidad se están suprimiendo sus talentos (Guerrero, 2006).

En contraparte, el poder utilizar diferentes espacios para aprender es una condición necesaria para este proceso. Los aprendizajes significativos permanentes y funcionales, se logran no solamente en el espacio físico del aula, sino también en todo lugar que ofrezca situaciones estimulantes que incrementen el interés por aprender ( $\mathrm{Fe}$ y Alegría del Perú, 2007).

Además, son numerosos los estudios que permiten llegar a la conclusión de que el coeficiente intelectual no es un determinante para el éxito, entendiendo éste como la adaptación e integración del individuo a la vida y la realización óptima de sus capacidades personales (Cabrera, 2006).

En las décadas de los años 70`s, David Ausubel creó una teoría denominada Teoría del Aprendizaje Significativo (Ausubel, 1978). De acuerdo a este tipo de aprendizaje, los nuevos conocimientos se incorporan en forma sustantiva en la estructura cognitiva del estudiante. Esto se logra cuando él o ella relaciona los nuevos conocimientos con los adquiridos anteriormente; pero también es necesario que se interesen por aprender lo que se le está mostrando (Maldonado, 2006).

La teoría destaca el hecho de que el aprendizaje sucede en una forma complicada, que requiere mucho más que la adquisición de hábitos y pequeñas habilidades. Los aprendices también tratan de desarrollar su intuición y tener nuevos elementos de juicio (DuBrin, 2003).

Precisamente todo el énfasis de la teoría se pone en el aprendizaje significativo, frente al memorístico. De esta manera se pueden utilizar con eficacia los conocimientos previos en la adquisición de nuevos conocimientos que, a su vez. El aprendizaje significativo sería el resultado de la interacción entre los conocimientos del que aprende y la nueva información que va a aprenderse (Ausubel, Novak, y Hanesian, 1983).

En la actualidad se está ante un nuevo paradigma de las inteligencias, cuyo modo de comprender la construcción y desarrollo del conocimiento ha sufrido un vertiginoso giro. Desde la perspectiva educativa, cada vez se argumenta más la 
multidimensionalidad y la complejidad, ya que la evolución de conocimientos y perspectivas se entrelazan en un cauce voraz (Picardo, 2002).

Sin embargo, los programas de enseñanza según Guerrero (2006), sólo se basan en las inteligencias lingüística y matemática, dando una mínima importancia a las otras. Es por ello que para lograr el objetivo de transformar a la escuela tradicional en una de Inteligencias Múltiples, se tiene que partir desde un trabajo en equipo en el que intervengan la escuela y el hogar

Es por esto que Gardner expresa que "una inteligencia implica la habilidad necesaria para resolver problemas o para elaborar productos que son de importancia en un contexto cultural o en una comunidad determinada" (Gardner: 1994, pág.11).

Gardner mencionado por Armstrong., expuso con relación a su teoría de las inteligencias múltiples: "desde mi punto de vista, la esencia de la teoría es respetar las múltiples diferencias que hay entre los individuos; las variaciones en la forma en que aparecen; los distintos modos por los cuales se pueden evaluar y el número casi infinito de modos en que estos pueden dejar una marca en el mundo" (Armstrong: 1999, pág.12).

Guerrero (2006), BuDrin (2003), Luca (2007), Armstrong (1999), Cabrera (2006), Lefrançois (1999), Wolfolk (1999) y Beltrán (1997) expresan que Howard Gardner añade que igual que hay muchos tipos de problemas que resolver, también hay muchos tipos de inteligencia:

- Inteligencia Lógica - Matemática, es la inteligencia que tienen los científicos. Se corresponde con el modo de pensamiento del hemisferio lógico y con lo que nuestra cultura ha considerado siempre como la única inteligencia.

- Inteligencia Lingüística, la que tienen los escritores, los poetas, los buenos redactores. Utiliza ambos hemisferios.

- Inteligencia Espacial, consiste en formar un modelo mental del mundo en tres dimensiones, es la inteligencia que tienen los marineros, los ingenieros, los cirujanos, los escultores, los arquitectos, o los decoradores.

- Inteligencia Musical es la capacidad de percibir, discriminar, transformar y expresar las formas musicales. Incluye la sensibilidad al ritmo, al tono y al timbre.

- Inteligencia Corporal - kinestésica, o la capacidad de utilizar el propio cuerpo para realizar actividades o resolver problemas. Es la inteligencia de las personas deportistas, artesanos, cirujanos y bailarines.

- Inteligencia Intrapersonal, es la que permite que uno se entienda a sí mismo. No está asociada a ninguna actividad concreta.

- Inteligencia Interpersonal, la que permite poder entender a los demás, y la se encuentra en los (as) buenos (as) profesionales en ventas, política, profesores o terapeutas.

- La inteligencia Inter e intrapersonal conforman la emocional y juntas determinan la capacidad de dirigir nuestra propia vida de manera satisfactoria.

- Inteligencia Naturalista, es la que se emplea cuando se observa y estudia la naturaleza. Es la que demuestran los biólogos o los herbolarios. 
Además, Gardner enfatiza el hecho de que todas las inteligencias son igualmente importantes. El problema es que el sistema escolar no las trata por igual y ha entronizado las dos primeras de la lista (la inteligencia lógico - matemática y la inteligencia lingüística), hasta el punto de negar la existencia de las demás (Guerrero, 2006).

Casi toda la experiencia en la medición de la inteligencia, ha sido en el área de las tareas matemáticas, lingüísticas y lógicas. Por lo tanto, esta idea de las inteligencias múltiples requiere la preparación de tareas nuevas que exploren las tareas antes ignoradas (Lefrançois, 1999).

Ya existen instituciones educativas que han incorporado la teoría de las inteligencias múltiples y luego de diez años de aplicación, han encontrado los siguientes beneficios: minimización de los problemas de conducta, aumento de la autoestima, desarrollo de la cooperación, incremento del número de líderes positivos, crecimiento del interés y afecto por la escuela y el estudio, presencia del constante humor, incremento del conocimiento en un $40 \%$ (Luca, 2007).

Basado en lo anterior, el propósito de esta investigación es aplicar un test que mida las distintas inteligencias múltiples en niños(as) de dos distintas escuelas, para así valorar si existe diferencia entre el modelo pedagógico academicista (enfoque tradicional) establecido por el Ministerio de Educación Pública y el modelo pedagógico cognitivo (enfoque constructivista).

\section{METODOLOGIA}

Sujetos: 29 niños y 20 niñas, con edades entre los 8 y 12 años, pertenecientes a dos distintas escuelas públicas de Heredia (Escuela Laboratorio y Escuela de San Isidro). Ellos estaban desde primer grado en su respectiva escuela.

Instrumentos y materiales: se aplicó un Test de Inteligencias Múltiples para niños(as) en edades escolares (Vega, 2006), el cual consta de quince ítems, subdivididos en siete categorías: lingüística, lógico-matemática, visual, sinestesia, musical, inter $\mathrm{e}$ intrapersonal. Los materiales que se utilizaron fueron: lápiz y borrador.

Procedimiento: se seleccionaron dos escuelas, una con un modelo pedagógico cognitivo (MPC) y otra con un modelo pedagógico academicista Luego, se confeccionaron cartas de autorización tanto para el centro educativo, como para los padres y madres de los (as) participantes del estudio. Además, se solicitó el permiso en cada centro educativo con la respectiva carta. Aprobado el ingreso y la aplicación del test, se coordinó por fechas la visita a cada centro educativo. Una vez en el centro, se seleccionaron los sujetos de acuerdo a su antigüedad en la institución y se les aplicó un test de inteligencias múltiples en la biblioteca.

Análisis Estadístico: como estadística inferencial se aplicó una ANOVA factorial de dos vías ( 2 x 2), todo corrido con el paquete estadístico SPSS para Windows, versión 8.0 . 


\section{Resultados}

No se encontró interacción significativa entre los factores estudiados $(\mathrm{F}: 0,167 ; \mathrm{p}>0,05)$ (ver gráfico 1). Por medio de la prueba ANOVA factorial de dos vías $(2 \times 2)$ se dieron diferencias significativas para la inteligencia lingüística $(F: 9,47 ; p<0,01)$ entre escuelas MPC (3,24 $\pm 1,24$ puntos) y academicista $(2,31 \pm 1,10$ puntos), asimismo se hallaron diferencias entre género ( $\mathrm{F}: 5,26 ; \mathrm{p}<0,05)$, mujeres $(3,25 \pm 1,02$ puntos) y hombres $(2,52 \pm 1,30$ puntos $)$.

Gráfico 1. Puntajes de la inteligencia verbal para hombres y mujeres tanto en la escuela academicista como en la MPC

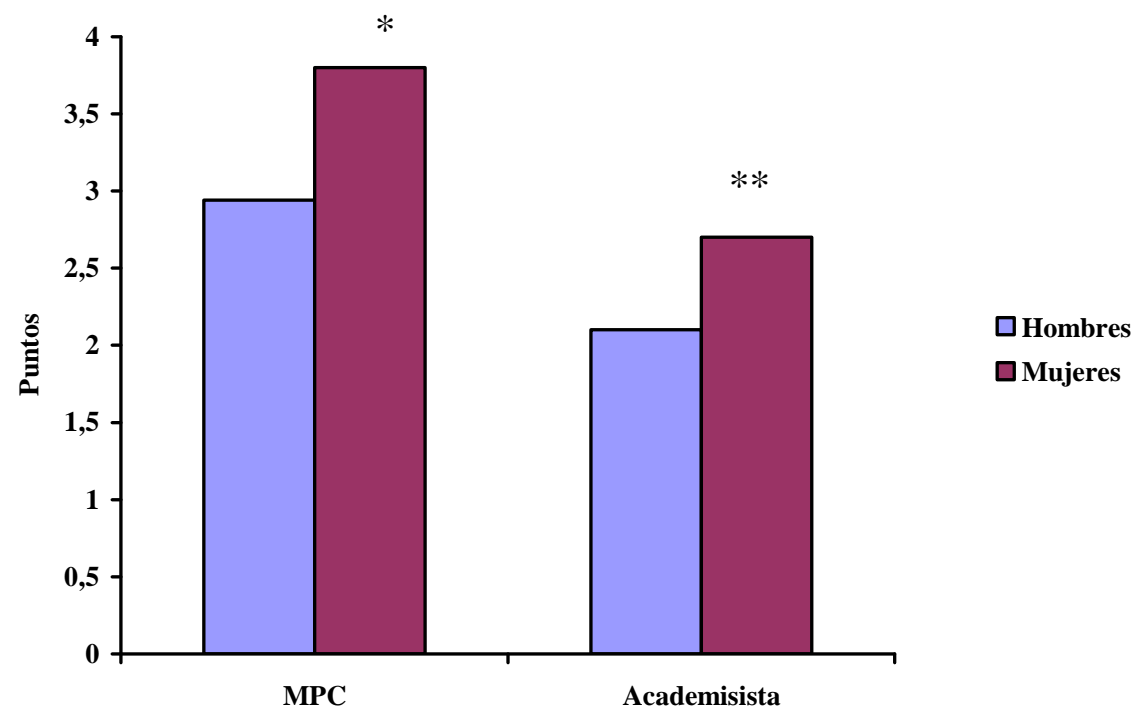

*F:9,47; $<<0,01$

$* * \mathrm{~F}: 5,26 ; \mathrm{p}<0,05$

Por medio de la prueba ANOVA factorial de dos vías $(2 \times 2)$ no se encontró interacción significativa entre género por escuelas $(\mathrm{F}: 0,079 ; \mathrm{p}>0,05)$, así mismo no se dieron diferencias significativas para la inteligencia musical $(\mathrm{F}: 0,81 ; \mathrm{p}>0,05)$ entre escuelas MPC $(3,13 \pm 1,18$ puntos) y academicista $(3,41 \pm 1,37$ puntos). Si se hallaron diferencias estadísticamente significativas entre género $(\mathrm{F}: 7,97 ; \mathrm{p}<0,05)$ (ver gráfico 2$)$.

Gráfico 2. Puntajes de la inteligencia musical para hombres y mujeres de ambas escuelas (MPC y academicista)

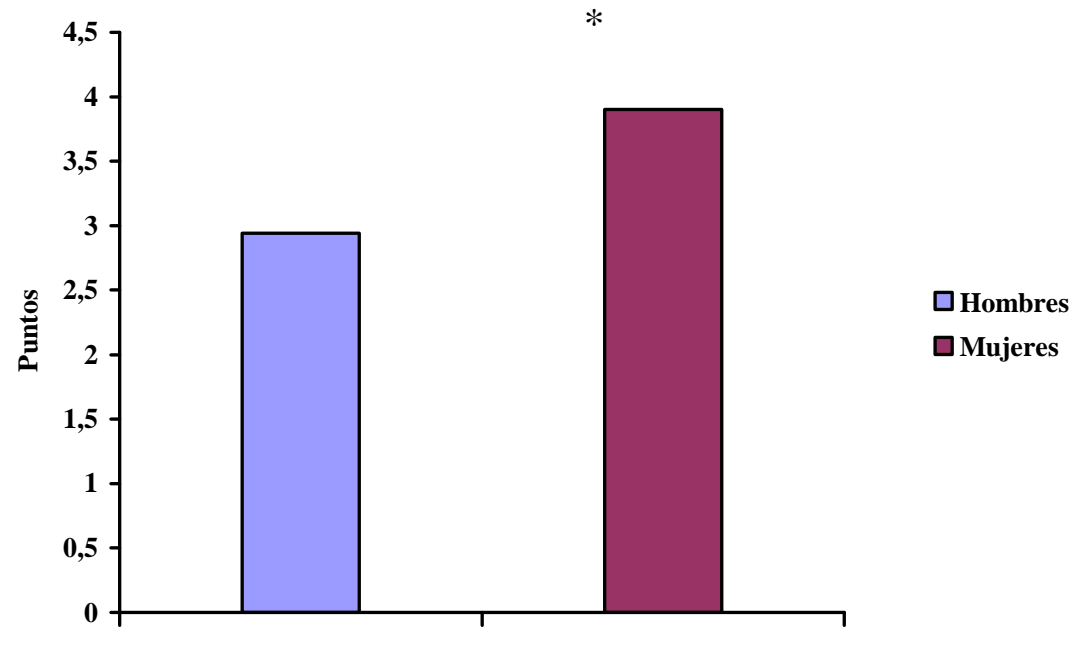


$* F: 7,97 ; p<0,05$

Utilizando la prueba ANOVA factorial de dos vías $(2 \times 2)$ no se encontró interacción significativa entre los factores estudiados ( $\mathrm{F}: 0,529 ; \mathrm{p}<0,05)$. Tampoco diferencias para la inteligencia lógico matemática $(\mathrm{F}: 0,80 ; \mathrm{p}>0,05)$ entre escuelas MPC $(3,55 \pm 1,32$ puntos $)$ y academicista $(3,73 \pm 0,87$ puntos $)$, así mismo no se establecieron diferencias entre género ( $\mathrm{F}: 2,29 ; \mathrm{p}>0,01)$, mujeres $(3,35 \pm 0,93$ puntos) y hombres $(3,81 \pm 1,18$ puntos). Tampoco se encontró interacción significativa entre los factores estudiados $(\mathrm{F}: 7,76 ; \mathrm{p}<0,05)$. Ni diferencias significativas para la inteligencia visual (F: 0,$50 ; p>0,05)$ entre escuelas MPC (3,03 $\pm 0,98$ puntos) y academicista $(2,75 \pm 1,27$ puntos), ni entre género ( $\mathrm{F}: 0,00 ; \mathrm{p}>0,01)$, mujeres $(2,89 \pm 1,07$ puntos) y hombres $(2,89 \pm 1,18$ puntos $)$.

No se encontraron diferencias significativas para la inteligencia cenestésica (F: 0,50; $\mathrm{p}>$ $0,05)$ entre escuelas, así mismo no hubo diferencia entre género ( $F: 0,00 ; p>0,01)$, mujeres $(2,89 \pm 1,07$ puntos) y hombres $(2,89 \pm 1,18$ puntos $)$. No se encontraron diferencias significativas para la inteligencia cenestésica $(\mathrm{F}: 0,50 ; \mathrm{p}>0,05)$ MPC $(3,03 \pm 0,98$ puntos $)$ y academicista $(2,75 \pm 1,27$ puntos $)$,

Al utilizar la prueba ANOVA factorial de dos vías $(2$ x 2) no se encontró interacción significativa entre los factores estudiados (F: 3,92; $\mathrm{p}<0,05)$. Ni diferencias significativas para la inteligencia intrapersonal (F: 0,$14 ; \mathrm{p}>0,05)$ entre escuelas MPC $(3,68 \pm 1,10$ puntos $)$ y academicista $(3,37 \pm 1,23$ puntos), así mismo no se encontraron diferencias entre sexo ( $F: 2,37 ; p>0,01)$, mujeres $(3,85 \pm 0,98)$ y hombres $(3,36 \pm 1,23)$.

Por medio de la prueba ANOVA factorial de dos vías ( 2 x 2) no se encontró interacción significativa entre los factores estudiados (F: 0,98; $\mathrm{p}<0,05)$. No se encontraron diferencias significativas para la inteligencia interpersonal $(\mathrm{F}: 4,38 ; \mathrm{p}>0,05)$ entre escuelas MPC $(4,10 \pm 0,77$ puntos) y academicista $(3,44 \pm 1,21$ puntos), así mismo no se encontraron diferencias entre sexo ( $\mathrm{F}: 4,38 ; \mathrm{p}>0,01$ puntos), mujeres $(4,15 \pm 0,81$ puntos) y hombres $(3,57 \pm 1,13$ puntos $)$.

\section{Discusión}

Se encontraron diferencias significativas en los puntajes de inteligencia lingüística entre escuelas MPC y academicista, por género. Según lo anterior, Luca (2007) menciona que los sistemas educativos con modelos pedagógicos tradicionales no son neutros, porque no prestan atención a todos los estilos de aprendizaje, ni valoran por igual todas las inteligencias o capacidades. En contraparte, Acosta (2002) expresa, que los nuevos modelos educativos con referencias pedagógicas y sociales han influido con aspectos renovadores produciendo cambios en los objetivos, programas y metodologías de todo el proceso enseñanza-aprendizaje, desde el campo educativo a través de la recreación y el juego. 
Además Novak y Gowin (1988) expresan que durante mucho tiempo se consideró que el aprendizaje era sinónimo de cambio de conducta, esto, porque dominó una perspectiva conductista de la labor educativa; sin embargo, se puede afirmar con certeza que el aprendizaje humano va más allá de ello, pues conduce a un cambio en el significado de la experiencia.

Asimismo, Ausubel (1983) menciona que el aprendizaje mecánico, contrariamente al significativo, se produce cuando no existen canales adecuados, de tal forma que la nueva información es almacenada arbitrariamente, sin interactuar con conocimientos pre-existentes, un ejemplo de ello sería el simple aprendizaje de fórmulas en física; esta nueva información es incorporada a la estructura cognitiva de manera literal y arbitraria puesto que consta de puras asociaciones, cuando él o la estudiante carece de conocimientos previos relevantes y necesarios para hacer que la tarea de aprendizaje sea potencialmente significativo.

La teoría de Ausubel (teoría del aprendizaje significativo) presta especial atención al aprendizaje verbal y, específicamente, al de conceptos. Es decir que, existe de tipo significativo cuando se relaciona intencionadamente material que es asociado con las ideas establecidas y pertinentes de la estructura cognitiva (Ausubel, Novak y Hanesian, 1983).

Además, en los puntajes de inteligencia musical, se encontraron diferencias estadísticamente significativas entre género, siendo las mujeres de ambas escuelas las que tuvieran mayor puntaje que los hombres. Chaves (2007) expresó que los resultados arrojados pueden deberse a un factor cultural debido más que todo a que en países de occidente, se nota más actitud machista. El género es una construcción socio-cultural que asigna determinados comportamientos a hombres y a mujeres, y que las diferencia en términos de papeles y actividades que desarrollan en la sociedad, va fortaleciendo jerarquías, entre unos y otras, es decir va estableciendo relaciones de poder y situaciones de inequidad entre ellos y ellas (Díaz 1999).

Además, las preguntas de éste test, para la inteligencia musical, parecen estar redactadas junto con una parte emocional. Es por esto que quizás las mujeres obtuvieran puntajes más altos que los hombres.

Según Chaves (2005) el niño y la niña al entrar en contacto con la cultura a la que pertenecen se apropian de los símbolos que son de origen social para posteriormente interiorizarlos. En este proceso, los infantes se adaptan de la cultura y construyen su identidad de género en las relaciones sociales, en la comunicación e interacción con la otredad.

Los primeros años de vida del ser humano son esenciales en su formación integral, puesto que el desarrollo de la inteligencia, la personalidad y el comportamiento social en las personas ocurre más rápido durante esos años (Rivero 1998).

Con respecto a la parte propiamente artística Parra (2006) menciona que el papel de la mujer en el terreno de la creatividad, ha estado muy escaso a lo largo de la historia, pero esto no solo se ha manifestado en este terreno, sino que también afecta al mundo de la 
pintura, la ciencia y otras artes creativas, la mujer tampoco ha ocupado un lugar de relevancia.

\section{Conclusiones}

- Los modelos pedagógicos enseñanza de los centros educativos públicos de Costa Rica deben actualizarse de acuerdo a las nuevas tendencias educativas (producto de los avances tecnológicos y de la globalización).

- Las inteligencias múltiples deberían ser más tomadas en cuenta dentro del planeamiento docente para así satisfacer con mayor calidad las demandas de la población estudiantil.

- El efecto de la sociedad y de la cultura pueden ser obstáculos para el desempeño ideal del niño dentro del contexto no solo educativo sino que podría marcar su vida como ser integral.

- Para futuras investigaciones relacionadas con inteligencias múltiples, se recomienda utilizar mayor población y de distintos niveles, no sólo de centros públicos sino también de centros privados, en donde se apliquen distintos modelos pedagógicos de enseñanza.

- Se recomienda utilizar otro tipo de test con mayor número de preguntas para cada una de las inteligencias.

- El Ministerio de Educación Pública debe hacer un análisis sobre los modelos pedagógicos de enseñanza, ya que el modelo que utiliza no está llenando las necesidades integrales de los infantes.

\section{BIBLIOGRAFÍA}

Acosta, L. (2002). La recreación: una estrategia para el aprendizaje. Colombia: Editorial Kinesis.

Armstrong, T. (1999). Las inteligencias múltiples en el aula. Argentina: Manantial.

Armstrong, T. (2001). Inteligencias múltiples: como descubrirlas y estimularlas en sus hijos. Editorial Norma.

Ausubel, D. (1978). Educational Psychology: A Cognitive View (2da. Edición). EUA: Rinehart \& Winston.

Beltrán, J. (1997). Psicología de la educación. México: Alfaomega.

Bolaños, G. y Molina, Z. (1990). Introducción al Currículo. San José: Editorial UNED.

Brenes, E. y Porras, M. (1994). Teoría de la Educación. San José, C.R.: Editorial UNED. 
Cabrera, A. (2006). Lengua, aprendizaje y enseñanza: el enfoque comunicativo: de la teoría a la práctica. Editorial Limusa.

Chaves, A. (2005). Las relaciones de género en el contexto escolar. Un estudio de caso a nivel de educación preescolar, Costa Rica. Instituto de Investigación para el Mejoramiento de la Educación Costarricense. Universidad de Costa Rica.

Chaves, C. (2007). (Comunicación personal, 14 de junio, 2007).

Díaz-Barriga, F. y Hernández, G. (2002). Estrategias docentes para un aprendizaje significativo. México: McGraw-Hill Interamericana.

Díaz, M. (1999). Algunas reflexiones acerca de: la dimensión de género en el curriculum de la educación parvularia. En TEMAS PEDAGÓGICOS. Serie de Cuadernillos de Estudio. Santiago de Chile: Junta Nacional de Jardines.

DuBrin, A. (2003). Fundamentos del comportamiento organizacional. México: Thomson Learning Ibero.

Flitner, W. (1972). Manual de Pedagogía General. Barcelona: Editorial Herder.

Fröbel, Federic. (1928). La educación del Hombre. Disponible en: http://www.cervantesvirtual.com/servlet/SirveObras/0159385243569594410225 7/index.htm. Fecha de acceso: 4 de julio, 2007.

Gardner, H. (1994). Estructuras de la mente. México: Fondo de Cultura Económica.

Guerrero, F. (2006). Inteligencias múltiples. Disponible en: http://www.monografias.com/trabajos12/intmult/intmult.shtml. Fecha de acceso: 20 de marzo, 2007.

Hernández, A. (1998). La preparación de formadores de profesores. Tomado de: Una Educación con calidad y equidad. España: Organización de Estado Iberoamericanos para la Educación, la Ciencia y la Cultura.

Incarbone, O. (2003). Del juego a la iniciación deportiva: 6 a 14 años. Argentina: Editorial STADIUM.

Lefrançois, G. (1999). El ciclo de la vida. Sexta Edición. México: Thomson Learning Ibero.

Luca, S. (2007). El docente y las inteligencias múltiples. Revista Iberoamericana de Educación. Disponible en: http://www.rieoei.org/deloslectores/616Luca.PDF. Fecha de acceso: 30 de abril, 2007.

Maldonado, M. (2006). El aprendizaje significativo de David Ausubel. Disponible en: http://www.monografias.com/trabajos10/dapa/dapa.shtml. Fecha de acceso: 15 de junio, 2007. 
Novak, J. y Gowin, B. (1988) Aprendiendo a Aprender. España: Martinez Roca.

Palma, V. (2005). La mujer y la música. Disponible en: http://www.webmujeractual.com/biografias/nombres/lamujerylamusica.htm. Fecha de acceso: 13 de junio, 2007.

Picardo, O. (2002). Educación y realidad: introducción a la filosofía del aprendizaje. Costa Rica: Impresora Obando S.A.

Rivero, J. (1998). La educación infantil en el siglo XXI. Proyecto Principal de Educación en América Latina y el Caribe. Boletín 47. Santiago de Chile: UNESCO, Oficina Regional de Educación para América Latina y el Caribe.

Rodríguez, M. y Muñoz, O. (2000). Los componentes académico y laboral e investigativo en el tratamiento de los conocimientos pedagógicos. Revista Digital. 5 (29). Disponible en: http://www.efdeportes.com/efd27a/investig.htm.

Ruiz, N. (2002). Estrategias y Métodos Pedagógicos. Colombia: Editorial H. Estefenn Prolibros y Cia. S. en C.

Vega, M. (2006). Test de inteligencias múltiples. Disponible en: http://www.rmm.cl/index_sub.php?id_contenido=3061\&id_portal=396\&id_secc ion=2560. Fecha de acceso: 10 de mayo, 2007.

Wolfolk, A. (1999). Psicología educativa. México: Prentice Hall.

Zapata, O. (1989). Juego y Aprendizaje Escolar. México: Editorial Pax México.

Fecha de recepción del artículo: 09 de junio del 2007.

Fecha de aceptación del artículo: 10 de julio del 2007.

Fecha de publicación del artículo: 31 de julio del 2007. 